\title{
A Stellar Model-fitting Pipeline for Solar-like Oscillations
}

\author{
T. S. Metcalfe*, O. L. Creevey ${ }^{\dagger}$ and J. Christensen-Dalsgaard** \\ ${ }^{*}$ High Altitude Observatory and Scientific Computing Division, NCAR, Boulder, Colorado, USA \\ $\dagger$ Instituto de Astrofísica de Canarias, La Laguna, Tenerife, Spain \\ ${ }^{* *}$ Department of Physics and Astronomy, Aarhus University, Aarhus, Denmark
}

\begin{abstract}
Over the past two decades, helioseismology has revolutionized our understanding of the interior structure and dynamics of the Sun. Asteroseismology will soon place this knowledge into a broader context by providing structural data for hundreds of Sun-like stars. Solar-like oscillations have already been detected from the ground in several stars, and NASA's Kepler mission is poised to unleash a flood of stellar pulsation data. Deriving reliable asteroseismic information from these observations demands a significant improvement in our analysis methods. We report the initial results of our efforts to develop an objective stellar model-fitting pipeline for asteroseismic data. The cornerstone of our automated approach is an optimization method using a parallel genetic algorithm. We describe the details of the pipeline and we present the initial application to Sunas-a-star data, yielding an optimal model that accurately reproduces the known solar properties.
\end{abstract}

Keywords: methods: numerical—stars: interiors—stars: oscillations

PACS: $97.10 . \mathrm{Sj}$

\section{THEORIST'S PERSPECTIVE}

In 2004, during the SOHO-GONG meeting at Yale University, Art Cox gave a talk summarizing the attempts to identify g-mode oscillations in the Sun. He put up a slide showing an image of the GOLF instrument on the SOHO satellite and said, "From a theorist's perspective, the light goes in here [pointing to the front end] and the answers come out here [pointing to the back end]". We all know that there are actually a few more steps involved when analyzing and interpreting real data, but this paper describes a computational method that attempts to bring the modeling of solar-like oscillations one step closer to Art's idealized picture.

In the past, ground-based data on solar-like oscillations in other stars have emerged slowly enough that we could try to model one star at a time. Beginning in October 2009, the Kepler mission promises to yield asteroseismic data for hundreds of stars every few months, so a hands-on approach will be a luxury we can no longer afford.

\section{COMPUTATIONAL METHOD}

The basic idea behind our model-fitting pipeline is frequencies in, stellar properties out. The pipeline takes as input the observed oscillation frequencies and other constraints from non-seismic data. We use the Aarhus stellar evolution code [ASTEC; 1] and the adiabatic pulsation code [ADIPLS; 2] coupled with a parallel genetic algorithm (GA) to identify a global match between the models and the observations. We use the result of the global search as the starting point for a local analysis, which employs a modified Levenberg-Marquardt (LM) algorithm with Singular Value Decomposition (SVD) to determine the final parameter values and uncertainties, and to probe the information content of the observational constraints. The output of the pipeline includes the mass, initial composition, mixing length and stellar age, as well as other properties of the optimal model such as the temperature, luminosity and radius.

\subsection{Global Search \& Local Analysis}

Since we are interested in developing a generalpurpose modeling tool for solar-like oscillations, we need to select a global method for optimizing the match between our model output and the available observations of any given star. Using only observations and the constitutive physics of the model to restrict the range of possible values for each parameter, a genetic algorithm [GA; $3,4]$ can provide a relatively efficient means of searching globally for the optimal model. Although it is more difficult for a GA to find precise values for the optimal set of parameters efficiently, it is well suited to search for the region of parameter space that contains the global minimum. In this sense, the GA is an objective means of obtaining a good first guess for a more traditional local analysis method, which can narrow in on the precise values and uncertainties of the optimal model parameters.

Our implementation of the GA optimizes four adjustable model parameters; these are the stellar mass $\left(M_{\star}\right)$ from 0.75 to $1.75 M_{\odot}$, the metallicity $(Z)$ from 0.002 to 0.05 (equally spaced in $\log Z$ ), the initial he- 
lium mass fraction $\left(Y_{0}\right)$ from 0.22 to 0.32 , and the mixing length parameter $(\alpha)$ from 1 to 3 . The stellar age $(\tau)$ is optimized internally during each model evaluation by matching the observed mean separation $\left\langle\Delta v_{0}\right\rangle$ between radial-mode frequencies (see section 2.2 ).

The GA uses two-digit decimal encoding, so there are 100 possible values for each parameter within the ranges specified above. Each run of the GA evolves a population of 128 models through 200 generations to find the optimal set of parameters, and we execute 4 independent runs with different random initialization to ensure that the best model identified is truly the global solution. This method requires about $10^{5}$ model evaluations, compared to $10^{8}$ models for a complete grid at the same sampling density, making the GA nearly 1000 times more efficient than a complete grid (currently 1 week of computing time, compared to many years for a grid). Of course, a grid could in principle be applied to hundreds of observational data sets without calculating additional models-but the GA approach also gives us the flexibility to improve the physical ingredients in the future, while the physics of a grid would be fixed.

Once the GA brings us close enough to the global solution, we can switch to a local optimization method. We implement a modified Levenberg-Marquardt (LM) algorithm that uses Singular Value Decomposition (SVD) to filter the least important information from the observables, some of which may be dominated by noise.

We have three main motivations for implementing a local optimization method at the end of the global search. First, the GA has a limited resolution for each parameter, and the values that match the observations best are most likely between the fixed sample points. The resolution of the local analysis is limited only by the precision of the stellar evolution and pulsation codes, so we use it to adjust the models below the resolution of the GA search. Second, we need to quantify the final parameter uncertainties and correlations (not provided by the GA), and we want to probe the information content of the observables to determine which future observations can potentially help the most. Third, the local analysis can explore the effects of using different physical descriptions of the stellar interior $[5,6]$. When the changes to the underlying physics are relatively subtle, we can assume that the global search by the GA using one set of assumptions will also provide a good starting point for a local analysis under slightly perturbed conditions.

\subsection{Fitting for Stellar Age}

During the optimization process, each model evaluation involves the computation of a stellar evolution track from the zero-age main sequence through a mass- dependent number of internal time steps, terminating prior to the beginning of the red-giant stage. Rather than calculate the pulsation frequencies for each of the 200300 models along the track, we exploit the fact that the average frequency spacing of consecutive radial overtones $\left\langle\Delta v_{0}\right\rangle$ in most cases is a monotonically decreasing function of age [7]. Once the evolution track is complete, we start with a pulsation analysis of the model at the middle time step and then use a binary decision tree-comparing the observed and calculated values of $\left\langle\Delta v_{0}\right\rangle$ - to select older or younger models along the track. In practice, this allows us to interpolate the age between the two nearest time steps by running the pulsation code on just 8 models from each evolution track.

\subsection{Correcting for Surface Effects}

The biggest challenge to comparing the oscillation frequencies from theoretical models with those actually observed in solar-type stars are the systematic errors due to surface effects. The mixing length parameterization of convection that is used in most stellar models is insufficient to describe the near-surface layers, and this leads to a systematic difference of several $\mu \mathrm{Hz}$ (up to about $0.3 \%$ for a solar model) between the observed and calculated frequencies (see Figure 1). The offset is nearly independent of the spherical degree $(l)$ of the mode and grows larger towards the acoustic cutoff frequency. The 3D simulations of convection that might in principle reduce this discrepancy for individual stars are far too computationally expensive for the model-fitting approach that we are developing. Instead, we adopt the method for empirical correction of surface effects described by Kjeldsen et al. [8], which uses the discrepancies between Model S and GOLF data for the Sun [9] to calibrate the empirical surface correction.

Following Kjeldsen et al. [8], we fit a power law to the differences between the frequencies of the radial modes of our fit to Model S and the corresponding frequencies from BiSON data [10] to characterize the surface effects. We found a power law exponent $b=4.82$, slightly lower than the value $(b=4.90)$ derived by Kjeldsen et al. using data from the GOLF experiment. With this exponent fixed, the recipe of Kjeldsen et al. describes how to predict the surface effect for any other set of calculated oscillation data, allowing us to apply this empirical correction to each of our models before comparing them to the observations. If our strategy of making this empirical correction to each of our models is to succeed, it must not only work well for models in a certain region of the search space-it must work best for a model that simultaneously matches all of the independent observational constraints within their uncertainties. 


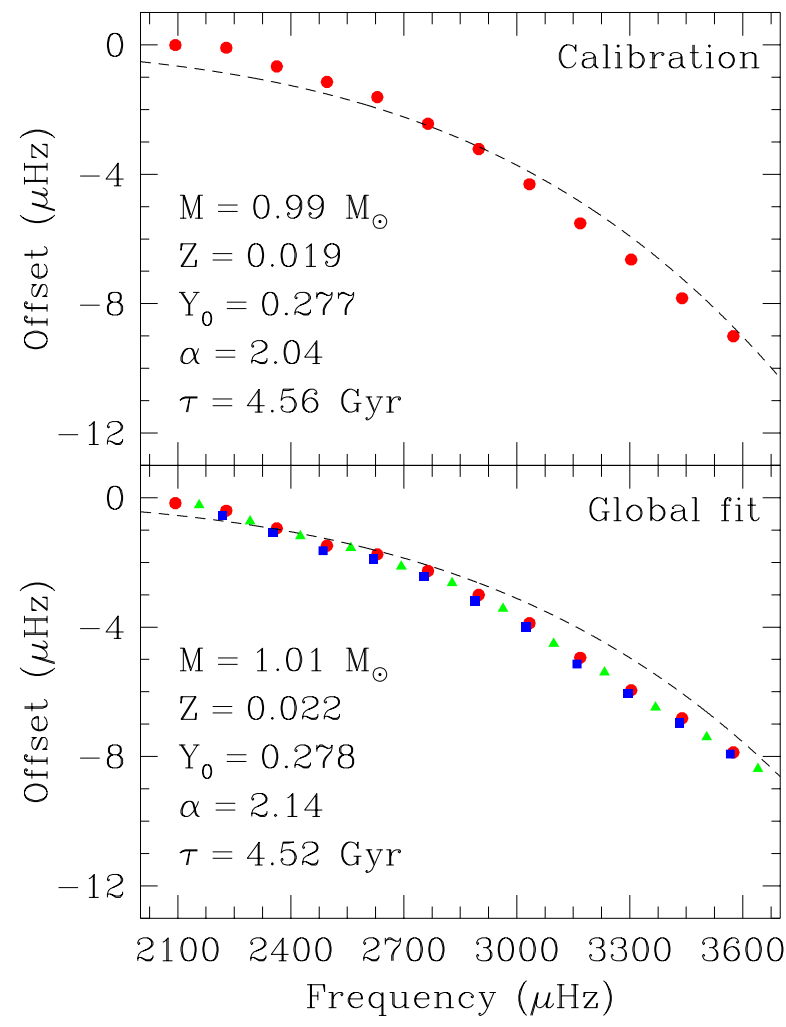

FIGURE 1. Top: Surface effects lead to systematic offsets between the radial modes (circles) from BiSON data and our fit to the Model S frequencies. Bottom: When we incorporate the resulting empirical correction into the models, applying it also to the dipole (triangles) and quadrupole (squares) modes, the global fit to the BiSON data reproduces the known solar properties within reasonable tolerances.

\section{INITIAL RESULTS}

Ultimately, our model-fitting pipeline can only be judged a success if it leads to accurate estimates of the stellar properties for the star that we know best: the Sun. There are many ingredients in our models that could in principle be insufficient descriptions of the actual conditions inside real stars-deficiencies that could easily lead to systematic errors in our determinations of the optimal model parameters for a given set of oscillation data. For example, we initially tried to use models that employed the simpler EFF equation of state [11] for computational expediency, but this led to estimates of the stellar mass about $10 \%$ too high for the Sun, and unacceptably large systematic errors on many of the other stellar properties. Even attempting to ignore the effects of helium settling proved to be too coarse an approximation, leading to 5\% errors on the mass. The only potential ingredient that we omitted without serious consequences was heavy element settling. This is not to say that simpler stellar mod- els cannot be used in the analysis of asteroseismic data, but rather that some of the more sophisticated ingredients are required to obtain accurate results from a global search of the parameter space.

After demonstrating the effectiveness of the method by fitting our models to synthetic data and calibrating the empirical surface correction using the differences between our fit to Model S and the BiSON data [see 12], we applied our model-fitting pipeline to solar data from the BiSON and GOLF experiments. The oscillation frequencies from these two sources are identical to each other within the observational uncertainties, but their noise properties are slightly different-allowing us to quantify any systematic errors that might arise from subtle effects in the data acquisition and analysis methods.

We assume that typical asteroseismic data from the Kepler mission will include twelve frequencies for each of the radial $(l=0)$, dipole $(l=1)$, and quadrupole $(l=$ 2 ) modes, with consecutive radial orders in the range $n=$ 14-25. Thus, we allowed the GA to fit a total of 36 oscillation frequencies. We assigned statistical uncertainties to each frequency by scaling up the errors on the corresponding modes by a factor of 10 , which is roughly what we expect from Kepler data $\left(\sigma_{v} \sim 0.1 \mu \mathrm{Hz}\right)$. We complemented this synthetic asteroseismic information with artificial data on the effective temperature and luminosity, with errors comparable to what is expected for stars in the Kepler Input Catalog [13, $T_{\text {eff }}=5777 \pm 100 \mathrm{~K}$, $\left.L_{\star} / L_{\odot}=1.00 \pm 0.1\right]$. The two sets of input data differed only in the absolute values of the oscillation frequencies (yielding distinct values of $\left\langle\Delta v_{0}\right\rangle$ for fitting the age), and in the statistical uncertainties assigned to each mode (leading to subtle differences in the weighting of the fit).

Both data sets led to identical values of the mass and metallicity from the global search, with slight variations in the values of the other parameters. These minor differences largely disappear after the local analysis. Note that because we multiplied the true observational errors by a factor of 10 for the fitting, the resulting values of $\chi_{\mathrm{R}}^{2}$ are $\sim 0.1$. Although the fits used a limited range of frequencies and did not include $l=3$ modes, the optimal models also match the modes with lower frequencies and higher degree (see the BiSON fit in Figure 2) and reproduce the known solar properties within reasonable tolerances.

\section{FUTURE WORK}

With the successful validation of our model-fitting pipeline using solar data, we now need to ensure that our adopted treatment of surface effects yields reasonable optimal models when applied to other stellar data. The obvious next step is to use archival ground-based data on several well studied solar-type stars to validate the pipeline for various stellar masses (e.g. $\alpha$ Cen A \& 

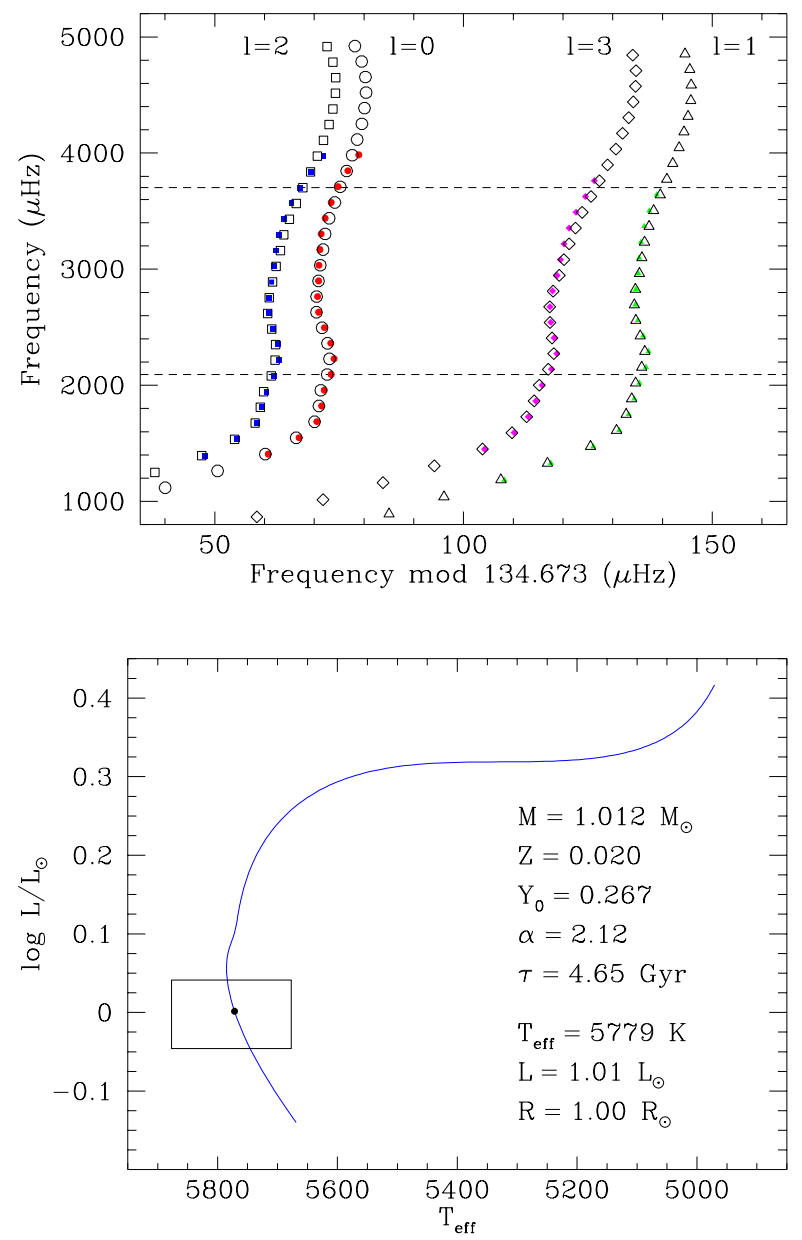

FIGURE 2. Top: An echelle diagram for the BiSON data (solid points), where we divide the oscillation spectrum into segments of length $\left\langle\Delta v_{0}\right\rangle$ and plot them against the oscillation frequency, along with the optimal model from our asteroseismic modeling pipeline (open points). Note that the pipeline only used the $l=0-2$ frequencies between the dashed lines for the fit, but the resulting optimal model also matches the $l=3$ modes and frequencies outside of the fitting range. Bottom: The evolution track (line) for the optimal model (solid point), which coincides with the middle of the observational error box (rectangle) defined by the temperature and luminosity constraints.

B, near $\sim 1.1$ and $\sim 0.9 M_{\odot}$ respectively) and for different evolutionary stages (e.g. the "future Sun" $\beta$ Hyi, at $\sim 7$ Gyr). Although Kjeldsen et al. [8] demonstrated their method by applying it to models of these three stars in addition to the Sun, our experience using it with solar data suggests that a global exploration of the models may present additional challenges.

Since our stellar models and the empirical correction for surface effects have both been calibrated using a main-sequence star at $1.0 M_{\odot}$, the $\alpha$ Cen system will help validate the models with interior physical conditions that differ slightly from those of the Sun.
Its proximity and multiple nature make it an excellent second test of our pipeline, because it has very well determined properties including stellar radii from interferometry [14]. There are also strong constraints on the component metallicities and effective temperatures [15], while the initial composition and age of the two stars are presumably identical. In the next phase of this project, we plan to use the published oscillation frequencies of $\alpha$ Cen A $[16,17,18]$ and $\alpha$ Cen B $[19,20]$ with the additional constraints from interferometry, spectroscopy, and the binary nature of the system to further validate our pipeline and the empirical correction for surface effects. Kjeldsen et al. [8] successfully applied their recipe to a set of stellar models that broadly resemble the components of the $\alpha$ Cen system, so we have good reason to believe that our implementation will also succeed-but this remains to be demonstrated.

The G2 subgiant $\beta$ Hyi has long been studied as a "future Sun", with an age near 7 Gyr. It has been characterized almost as extensively as the $\alpha$ Cen system, including recent interferometric measurements of its diameter [21] and dual-site asteroseismic observations that determined its mean density with an accuracy of $0.6 \%$ [22]. These data included the detection of several $l=1$ modes that deviate from the asymptotic frequency spacing, suggesting that they are "mixed modes" behaving like g-modes in the core and p-modes in the envelope. This is expected for evolved stars like $\beta$ Hyi because as they expand and cool the p-mode frequencies decrease, while the g-mode frequencies increase as the star becomes more centrally condensed. This leads to a range of frequencies where these modes can overlap and exchange their character, manifested as so-called avoided crossings. This behavior changes very quickly with stellar age, and propagates from one mode to the next as a star continues to evolve. Consequently, the particular mode affected yields a very strong constraint on the age of the star [see 23]. In subsequent work, we plan to use the published oscillation data for $\beta$ Hyi [22] along with the constraints from interferometry and spectroscopy to validate our pipeline and the empirical treatment of surface effects for stars that are significantly more evolved than the Sun. This will require an automated method to recognize mixed modes in the data set and to incorporate them into the optimization of stellar age along each track.

Once we have validated the model-fitting pipeline with additional stars that sample a range of masses and evolutionary stages, we can begin to consider additional observables and parameters that are not constrained by currently available data sets. High-quality asteroseismic data are soon expected from the Kepler mission, spanning sufficiently long periods of time that the effects of rotation [24, 25, 26] and magnetic activity cycles [27, 28] should be detectable. The Kepler mission is designed to discover Earth-sized habitable planets, and our model- 
fitting pipeline will be able to characterize the planethosting stars with asteroseismology. This is essential to convert precise transit photometry into an absolute radius for the planetary body. In addition, accurate rotation rates and ages will provide clues about the formation and evolution of the planet-hosting systems. The determination of accurate stellar properties for a broad array of solartype stars will give us a new window on stellar structure and evolution, and will provide a broader context for our understanding of the Sun and our own solar system. We hope to facilitate this process by applying our stellar model-fitting pipeline to the data that will soon emerge from the Kepler mission.

\section{ACKNOWLEDGMENTS}

The authors wish to thank Tim Brown and Margarida Cunha for helpful discussions during the early phases of this project. This work was supported in part by an NSF Astronomy \& Astrophysics Fellowship (to T.S.M.) under award AST-0401441, by a Newkirk Graduate Fellowship (to O.L.C.) at the High Altitude Observatory and by the European Helio- and Asteroseismology Network (HELAS), a major international collaboration funded by the European Commission's Sixth Framework Programme, by the Danish Natural Science Research Council, and by NASA grant NNX09AE59G. Computer time was provided by NSF MRI grants CNS-0421498, CNS0420873, CNS-0420985, the University of Colorado, and a grant from the IBM Shared University Research (SUR) program. The National Center for Atmospheric Research is a federally funded research and development center sponsored by the U.S. National Science Foundation.

\section{REFERENCES}

1. J. Christensen-Dalsgaard, Astrophysics \& Space Science, 316, 13-24 (2008).

2. J. Christensen-Dalsgaard, Astrophysics \& Space Science, 316, 113-120 (2008).

3. P. Charbonneau, Astrophysical Journal Supplement, 101, 309-344 (1995).

4. T. S. Metcalfe, and P. Charbonneau, Journal of Computational Physics, 185, 176-193 (2003).

5. O. L. Creevey, PhD thesis, University of La Laguna (2008).

6. O. L. Creevey, "Testing the potential and limitations of seismic data," in ASP Conf. Ser., 2009, arXiv:0810.2442.

7. J. Christensen-Dalsgaard, "On the Asteroseismic HR Diagram," in ASP Conf. Ser. 42: GONG 1992. Seismic Investigation of the Sun and Stars, edited by T. M. Brown, 1993, pp. 347-350.

8. H. Kjeldsen, T. R. Bedding, and J. Christensen-Dalsgaard, Astrophysical Journal Letters, 683, L175-L178 (2008).
9. M. Lazrek, F. Baudin, L. Bertello, P. Boumier, J. Charra, D. Fierry-Fraillon, E. Fossat, A. H. Gabriel, R. A. García, B. Gelly, C. Gouiffes, G. Grec, P. L. Pallé, F. Pérez Hernández, C. Régulo, C. Renaud, J.-M. Robillot, T. Roca Cortés, S. Turck-Chièze, and R. K. Ulrich, Solar Physics, 175, 227-246 (1997).

10. W. J. Chaplin, Y. Elsworth, G. R. Isaak, B. A. Miller, and R. New, Monthly Notices of the Royal Astronomical Society, 308, 424-430 (1999).

11. P. P. Eggleton, J. Faulkner, and B. P. Flannery, Astronomy \& Astrophysics, 23, 325-330 (1973).

12. T. S. Metcalfe, O. L. Creevey, and J. ChristensenDalsgaard, Astrophysical Journal, 699, 373-382 (2009).

13. D. W. Latham, T. M. Brown, D. G. Monet, M. Everett, G. A. Esquerdo, and C. W. Hergenrother, Bulletin of the American Astronomical Society 37, 1340 (2005).

14. P. Kervella, F. Thévenin, D. Ségransan, G. Berthomieu, B. Lopez, P. Morel, and J. Provost, Astronomy \& Astrophysics, 404, 1087-1097 (2003).

15. G. F. Porto de Mello, W. Lyra, and G. R. Keller, Astronomy \& Astrophysics, 488, 653-666 (2008).

16. F. Bouchy, and F. Carrier, Astronomy \& Astrophysics, 390, 205-212 (2002).

17. T. R. Bedding, H. Kjeldsen, R. P. Butler, C. McCarthy, G. W. Marcy, S. J. O'Toole, C. G. Tinney, and J. T. Wright, Astrophysical Journal, 614, 380-385 (2004).

18. M. Bazot, F. Bouchy, H. Kjeldsen, S. Charpinet, M. Laymand, and S. Vauclair, Astronomy \& Astrophysics, 470, 295-302 (2007).

19. F. Carrier, and G. Bourban, Astronomy \& Astrophysics, 406, L23-L26 (2003).

20. H. Kjeldsen, T. R. Bedding, R. P. Butler, J. ChristensenDalsgaard, L. L. Kiss, C. McCarthy, G. W. Marcy, C. G. Tinney, and J. T. Wright, Astrophysical Journal, 635, 1281-1290 (2005).

21. J. R. North, J. Davis, T. R. Bedding, M. J. Ireland, A. P. Jacob, J. O'Byrne, S. M. Owens, J. G. Robertson, W. J. Tango, and P. G. Tuthill, Monthly Notices of the Royal Astronomical Society, 380, L80-L83 (2007).

22. T. R. Bedding, H. Kjeldsen, T. Arentoft, F. Bouchy, J. Brandbyge, B. J. Brewer, R. P. Butler, J. ChristensenDalsgaard, T. Dall, S. Frandsen, C. Karoff, L. L. Kiss, M. J. P. F. G. Monteiro, F. P. Pijpers, T. C. Teixeira, C. G. Tinney, I. K. Baldry, F. Carrier, and S. J. O'Toole, Astrophysical Journal, 663, 1315-1324 (2007).

23. J. Christensen-Dalsgaard, Solar Physics, 220, 137-168 (2004).

24. L. Gizon, and S. K. Solanki, Astrophysical Journal, 589, 1009-1019 (2003).

25. J. Ballot, R. A. García, and P. Lambert, Monthly Notices of the Royal Astronomical Society, 369, 1281-1286 (2006).

26. J. Ballot, T. Appourchaux, T. Toutain, and M. Guittet, Astronomy \& Astrophysics, 486, 867-875 (2008).

27. W. J. Chaplin, Y. Elsworth, G. Houdek, and R. New, Monthly Notices of the Royal Astronomical Society, 377, 17-29 (2007).

28. T. S. Metcalfe, W. A. Dziembowski, P. G. Judge, and M. Snow, Monthly Notices of the Royal Astronomical Society, 379, L16-L20 (2007). 